\title{
Prediction of Hysteresis Associated with the Static Stall of an Airfoil
}

\author{
S. Mittal* and P. Saxena ${ }^{\dagger}$ \\ Indian Institute of Technology, Kanpur 208016 , India
}

\section{Introduction}

7 HE basic phenomenon of stall associated with airfoils is quite 1 well understood and has now become standard textbook material. It is caused by massive flow separation resulting in sharp drop in lift and increase in the drag acting on the airfoil. In certain cases, hysteresis in the flow has been observed for angles of attack close to the stall angle. However, this phenomenon is not very well understood. Hoffmann' has reported the hysteresis loop in the data for aerodynamic coefficients for a NACA 0015 airfoil. He also studied the effect of freestream turbulence (FST) on the performance characteristics of the airfoil. The hysteresis in the data can be observed for low FST but disappears for high FST. The present work is an effort to study the behavior of the flow near stall by solving the governing flow equations numerically. Carefully conducted computations are utilized to track the hysteresis loop in the aerodynamic data close to the stall angle. To the best of the knowledge of these authors, this is the first effort of its nature. The incompressible, Reynoldsaveraged Navier-Stokes (RANS) equations, in conjunction with the Baldwin-Lomax model ${ }^{2}$ for turbulence closure are solved using stabilized finite element formulations. The finite element mesh consists of a structured mesh close to the body and an unstructured part, generated via Delaunay's triangulation, away from the body. This type of a grid has the ability of handling fairly complex geometries while still providing the desired resolution close to the body to effectively capture the boundary-layer flow, especially in the context of unsteady flows. Despite the simplicity of the Baldwin-Lomax model, its implementation with unstructured grids is not trivial. The interested reader is referred to the articles by Kallinderis ${ }^{3}$ and Mavriplis ${ }^{4}$ for details.

The finite element formulations and their implementations used in the present work are well proven and have been utilized to

Received 2 August 1999; revision received 18 November 1999; accepted for publication 15 December 1999. Copyright (C) 2000 by the American Institute of Aeronautics and Astronautics, Inc. All rights reserved.

*Associate Professor, Department of Aerospace Engineering; smittal@ iitk.ac.in.

${ }^{\dagger}$ Graduate Student. Debartment of Aerospace Engineering. 
solve a variety of unsteady flow problems. ${ }^{5}$ To stabilize the computations against spurious numerical oscillations in advectiondominated flows and to enable the use of equal-order-interpolation velocity-pressure elements, streamline-upwind/Petrov-Galerkin and pressure-stabilizing/Petrov-Galerkin stabilization techniques ${ }^{5}$ are employed. In this technique, stabilizing terms that are based on the element level integrals of the residuals are added to the basic Galerkin formulation. The time integration of the equations is carried out via the time-accurate version of the generalized trapezoidal rule (Crank-Nicholson). The nonlinear, implicit equation systems resulting from the finite element discretization of the flow equations are solved using the generalized minimal residual technique in conjunction with diagonal preconditioners.

\section{Results and Discussion}

The NACA 0012 airfoil resides in a rectangular computational domain whose upstream and downstream boundaries are located at 5 and 11 chord lengths from the leading edge, respectively. The upper and lower boundaries are placed at five chord lengths each from the leading edge. The no-slip condition is specified for the velocity on the airfoil surface, and freestream values are assigned for the velocity at the upstream boundary. At the downstream boundary a Neumann-type boundary condition for the velocity is specified that corresponds to zero viscous stress vector. On the upper and lower surface boundaries the component of velocity normal to the component of stress vector along these boundaries is prescribed zero value. The Reynolds number based on the chord length of the airfoil, freestream velocity and viscosity of the fluid is $10^{6}$

Figure 1 shows the close-up view of a typical finite element mesh employed for the computations. Computations for $\alpha=0,5,10$, and $15 \mathrm{deg}$ are initiated with the steady-state solution at the respective angles of attack for $\operatorname{Re}=10^{2}$. The steady-state solution is obtained by simply removing the unsteady terms from the governing equations. This is possible because the present method utilizes an implicit time-integrating procedure. The Reynolds number is then ramped up to $10^{6}$ over a period of approximately 500 time steps and the turbulence model is turned on. The computations are continued until the fully developed flow is obtained. The fully developed solution for $\alpha=15 \mathrm{deg}$ is used as an initial condition for computing the flow for $\alpha=16 \mathrm{deg}$. The finite element mesh for this computation is obtained from the mesh for $\alpha=15 \mathrm{deg}$ via a mesh-moving scheme that preserves the number of nodes and their connectivity. Once the fully developed flow for $\alpha=16 \mathrm{deg}$ is obtained it is utilized as an initial condition to obtain the flow for $\alpha=17 \mathrm{deg}$. The process continues until the fully developed flow for $\alpha=20$ deg is computed. The series of solutions at various angles of attack, obtained by this method, approximates the situation where the wind-tunnel data for a static airfoil are collected by increasing the angle of attack in small increments. Our computations indicate that the flow ceases to be steady beyond $\alpha=17 \mathrm{deg}$ and vortex shedding is observed. The unsteadiness in the flow increases with the angle of attack. Computations have also been carried out for the $\alpha=20$ deg case by using, as the initial condition, the steady-state solution for the same $\alpha$ and $\operatorname{Re}=10^{2}$. It is found that the fully developed unsteady solutions obtained by starting from these two different initial conditions are the same. Next, the solution for $\alpha=20 \mathrm{deg}$ is used as an

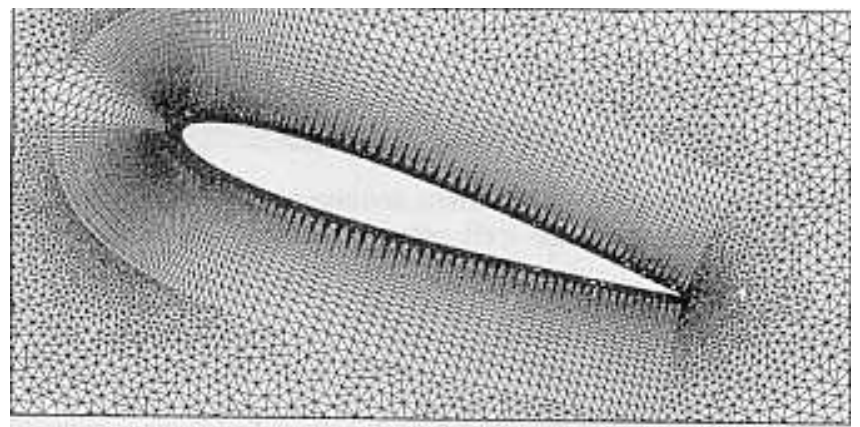

Fig. $1 R e=10^{6}$ flow past a NACA 0012 airfoil at 18-deg angle of attack; a close-up view of the finite element mesh; 19,071 nodes, 37,896 elements.
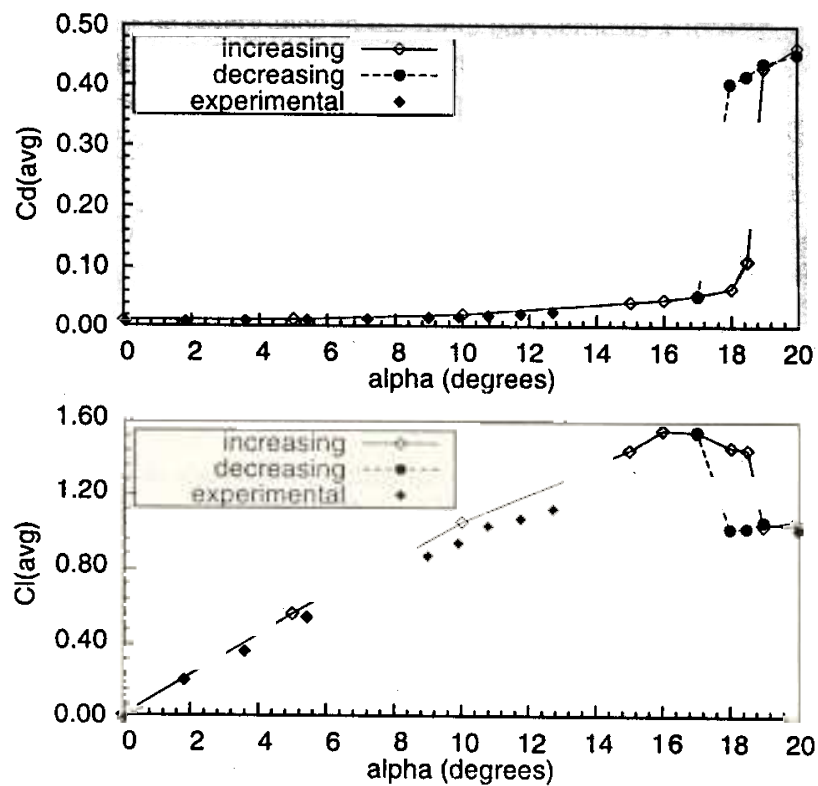

Fig. $2 R e=10^{6}$ flow past a NACA 0012 airfoil: variation of the timeaveraged drag and lift coefficients with angle of attack; hysteresis can be observed near the stall angle; the experimental results are from Ref. 6 for Mach $=0.3$ flow and $R e=1.8 \times 10^{6}$
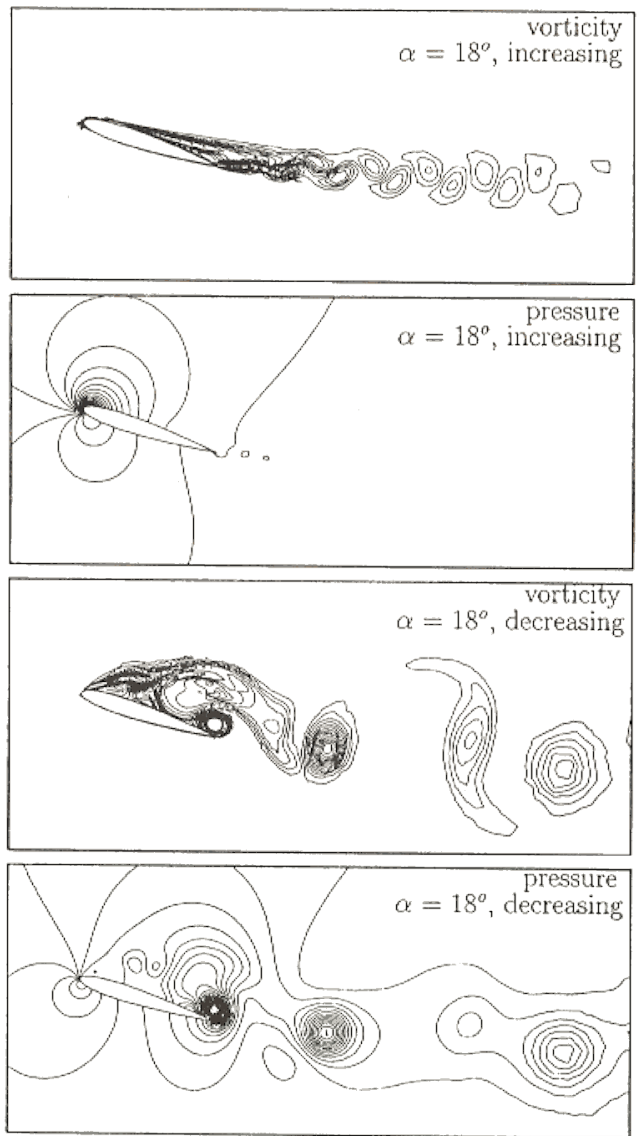

Fig. $3 R e=10^{6}$ flow past a NACA 0012 airfoil at 18-deg angle of attack: vorticity and pressure fields for the computed solutions obtained with increasing and decreasing angle of attack.

initial condition to obtain the solution for $\alpha=19 \mathrm{deg}$, which is in turn utilized to compute the flow for $\alpha=18 \mathrm{deg}$, and so on. The solutions obtained in this manner will be referred to as the decreasing angle solutions. Figure 2 shows the variation of the time-averaged values of the aerodynamic coefficients with the angle of attack for the computed solutions along with some experimental results. ${ }^{6}$ The experimental results correspond to Mach $=0.3$ and $R e=1.8 \times 10^{6}$ flow. In the experiments the transition point was not fixed and no 
attempt was made to determine its location. However, the present computations do not model transition and the flow is assumed to be turbulent right at the leading edge of the airfoil. Additionally, the flow is incompressible and the Reynolds number is a little lower than that in the experiments. These reasons might explain some of the differences that can be noticed between the numerical and experimental results. Hysteresis in the aerodynamic coefficients for the computed solution can be observed for $\alpha$ lying between 17 and 19 deg. Qualitatively, these results look similar to those reported by Hoffman' for his experiments with the NACA 0015 airfoil for low FST.

The hysteretic behavior of the flow is caused by its ability to remember its past history. The starting point for the flows along the increasing angle branch is an attached flow, whereas it is a massively separated flow for the flows along the branch corresponding to the decreasing angle. The instantaneous vorticity and pressure fields for the two solutions for $\alpha=18 \mathrm{deg}$, in the fully developed state, are shown in Fig. 3. The solution along the increasing angle branch shows relatively low unsteadiness compared to the one that has been obtained with the decreasing angle of attack. This is also reflected in the time histories of the lift and drag coefficients for the two solutions (not shown here). The unsteady component of the aerodynamic coefficients for the flow with decreasing angle is significantly larger than that with the increasing angle. Because both of the computations have been carried out with identical finite element meshes, it can be concluded that it is the initial condition that is responsible for the multiplicity of solution.

\section{Conclusion}

Results have been presented for computation of flow past a NACA 0012 airfoil using RANS equations in conjunction with a BaldwinLomax turbulence model for closure. A well-proven stabilized finite element method that has been applied to various flow problems earlier has been utilized to solve the incompressible Navier-Stokes equations in the primitive variables formulation. Hysteresis in the flow has been observed for angles of attack close to the stall angles of the airfoil. The ability of the flow to remember its past history is responsible for its hysteretic behavior. For the same angle of attack, the flow obtained with the increasing angle results in an almost attached flow with higher lift and lower drag, whereas the one with decreasing angle of attack is associated with large unsteadiness, lower lift, and higher drag. These computations demonstrate that a simple turbulence model in conjunction with an accurate flow solver can replicate fairly complex physical phenomenon. The success of the turbulent flow calculations depend not only on the complexity of the turbulence model, but also on the accuracy of the underlying basic numerical scheme for solving RANS.

\section{Acknowledgments}

Partial support for this work has come from the Aeronautical Research and Development Board, India, under Project ARDB-AE970310 with the Department of Aerospace Engineering, India, Institute of Technology, Kanpur.

\section{References}

${ }^{1}$ Hoffmann, J. A., "Effects of Freestream Turbulence on the Performance Characteristics of an Airfoil," AIAA Journal, Vol. 29, No. 9, 1991, pp. 1353, 1354.

${ }^{2}$ Baldwin, B., and Lomax., H., "Thin Layer Approximation and Algebraic Turbulence Model for Separated Turbulent Flows," AIAA Paper 78-257, Jan. 1978.

${ }^{3}$ Kallinderis, Y., "Algebraic Turbulence Modeling for Adaptive Unstructured Grids," AIAA Journal, Vol. 30, No. 3, 1992, pp. 631-639.

${ }^{4}$ Mavriplis, D. J., "Turbulent Flow Calculations Using Unstructured and Adaptive Meshes," International Journal for Numerical Methods in Fluids, Vol. 13, No. 9, 1991, pp. 1131-1152.

${ }^{5}$ Mittal, S., Kumar, V., and Raghuvanshi, A., "Unsteady Incompressible Flow Past Two Cylinders in Tandem and Staggered Arrangements," International Journal for Numerical Methods in Fluids, Vol. 25, No. 11, 1997, pp. 1315-1344.

${ }^{6}$ Thibert, J. J., Grandjacques, M., and Ohman, L. H., "Experimental Database for Computer Program Assessment," TR AR-138, AGARD, May 1979.

J. Kallinderis Associate Editor

\section{On Mixing Enhancement via Nozzle Trailing-Edge Modifications in High-Speed Jets}

\author{
J.-H. Kim* and M. Samimy ${ }^{\dagger}$ \\ Ohio State University, Columbus, Ohio 43210
}

\section{Introduction}

$\mathbf{E}$ XTENSIVE research has been conducted over the past few years on mixing enhancement using trailing-edge modifications in supersonic rectangular jets. The trailing-edge modifications (or cutouts) were either on the splitter plate in a half nozzle ${ }^{1}$ or on the nozzle extension in a full nozzle. ${ }^{2}$ The use of trailing-edge modifications of this type resulted in significant mixing enhancement in the underexpanded flow regime, moderate enhancement in the overexpanded regime, and no significant mixing enhancement in the ideally expanded flow regime. ${ }^{1,2}$ Note that the mixing enhancement was achieved without thrust loss in these experiments. Through a detailed investigation of the physics of the vortex generation mechanism, Kim and Samimy ${ }^{2}$ concluded that the spanwise pressure gradient on the modified trailing-edge surfaces is the major source of streamwise vorticity.

The reason for the use of the trailing-edge modifications on the splitter plate or on the nozzle extension in a full nozzle was to simplify the problem so that the physics of the streamwise vorticity generation mechanism could be identified. However, in the practical applications, the cutouts would be located on the nozzle blocks, that is, before the expansion in the nozzle diverging section is completed. The purpose of the present experiments is to show that a cutout on the contoured nozzle block is effective in mixing enhancement in all flow regimes, including the ideally expanded regime.

\section{Experimental Facility and Techniques}

The air delivery system to the nozzle is similar to the one used by Kim and Samimy ${ }^{2}$ with additional flow conditioning screens installed in the stagnation chamber. ${ }^{3}$ As in the previous experiments, ${ }^{2}$ the nozzle exit measures $2.86 \mathrm{~cm}$ wide and $0.95 \mathrm{~cm}$ high, with an equivalent diameter $\left[D_{\mathrm{eq}}=\left(4 A_{\mathrm{exit}} / \pi\right)^{1 / 2}\right]$ of $1.86 \mathrm{~cm}$. The schematic of the baseline nozzle and the types of cutouts on the nozzle block are shown in Fig. 1. Contrary to the previous trailingedge modifications, in which the cutouts were either on the splitter plate $^{1}$ or on the nozzle extension in a full nozzle, ${ }^{2}$ the cutouts are located on the contoured nozzle blocks. These contoured nozzles are designed to generate uniform flows at the nozzle exit, which operated at the design conditions. The nozzle block has a cutout of either rectangular type on the side (RS), shown in Fig. 1b, or rectangular type at the center (RC), shown in Fig. 1c. The cutouts on the nozzle block are more representative of practical applications. This is the only major difference between the present nozzles and the previous nozzles. The cutout dimensions are the same as those used in the previous experiments. ${ }^{1,2}$ The wall thickness at the cutout edge in the present case is gradually decreased from $4.5 \mathrm{~mm}$ at the beginning of the cutout to $1 \mathrm{~mm}$ at the nozzle exit, whereas it was $1 \mathrm{~mm}$ in the previous cases.

As in previous work, the instantaneous cross-sectional images were acquired by the laser sheet illumination technique. ${ }^{1,2}$ The visualizations of the jet cross section were performed at four downstream locations, that is, $x / D_{\mathrm{eq}}=1,2,4$, and 8 . The jet was operated at three fully expanded jet Mach numbers of 1.75,2.0 (design Mach number), and 2.5 .

Received 26 October 1999; revision received 22 December 1999; accepted for publication 27 December 1999. Copyright (C) 2000 by the American Institute of Aeronautics and Astronautics, Inc. All rights reserved.

${ }^{*}$ Postdoctoral Researcher, Department of Mechanical Engineering. Member AIAA.

${ }^{\dagger}$ Professor and Associate Chairman, Department of Mechanical Engineering. Associate Fellow AIAA. 


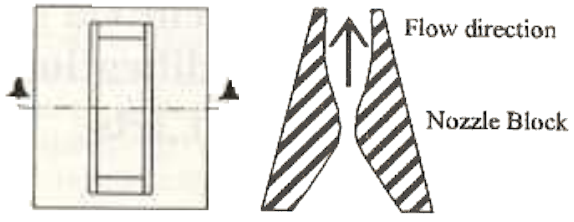

a) Side and end views of the baseline nozzle

b) RS

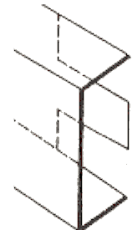

c) $\mathrm{RC}$

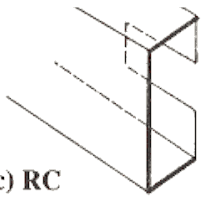

Fig. 1 Schematic of nozzle configurations.

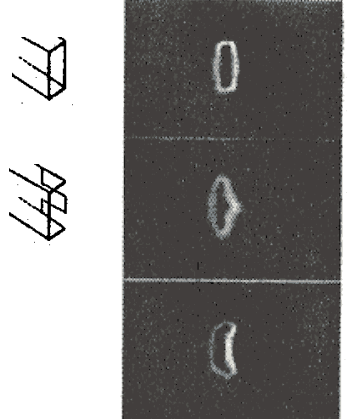

$\mathrm{M}_{\mathrm{j}}=1.75$

a) $x / D_{\mathrm{eq}}=2$

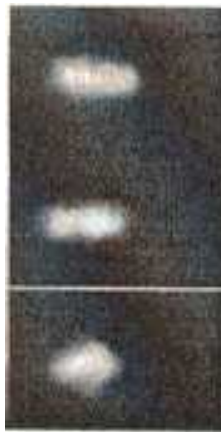

$\mathrm{M}_{\mathrm{j}}=\mathbf{1 . 7 5}$

b) $x / D_{\mathrm{eq}}=8$

Fig. 2 Average cross-sectional images; physical dimensions of each image are a) $116.4 \mathrm{~mm}\left(6.3 D_{\text {eq }}\right)$ wide and $77.6 \mathrm{~mm}\left(4.2 D_{\text {eq }}\right)$ high and b) $178.5 \mathrm{~mm}\left(9.6 D_{\mathrm{eq}}\right)$ wide and $119.0 \mathrm{~mm}\left(6.4 D_{\mathrm{eq}}\right)$ high.

\section{Results}

From the average and instantaneous jet cross-sectional images at four downstream locations, the jet evolution and overall mixing were investigated. As in the previous experiments, ${ }^{1.2}$ the pair of counterrotating streamwise vortices generated by each cutout dictate the cross-sectional development and mixing characteristics of the jet. The mixing area at a given downstream location was calculated and normalized with a reference mixing area. ${ }^{1,2}$ The reference mixing area for each nozzle was acquired at $M_{j}=2.0$ (ideally expanded) using the baseline nozzle after flow visualizations for the nozzle operated at three flow conditions were performed. The average jet cross-sectional images of 50 instantaneous frames at $x / D_{\mathrm{eq}}=2$ and 8 are shown in Fig. 2. The image distortions due to the angle between the camera axis and the jet axis were corrected using image processing.

\section{Underexpanded Case $\left(M_{j}=2.5\right)$}

As shown in Fig. 2 and discussed in the previous experiments, ${ }^{2}$ two types of counter-rotating streamwise vortices were observed in this flow regime: a kidney type by nozzle RS and a mushroom type by nozzle RC. It is believed that the spanwise pressure gradient on the nozzle block surface around the cutout is the major source of streamwise vorticity in the underexpanded case. In the underexpanded flow regime for the base nozzle, the effects of corner vortices can be easily seen in Fig. 2 (Ref. 1).

The jet cross-sectional development for nozzles with cutouts on the contoured nozzle block is quite similar to that for nozzles with cutouts on the splitter plate ${ }^{1}$ or on the nozzle extension. ${ }^{2}$ Even though $M_{j}$ is the same in this and the previous experiments, the degree of underexpansion in the present case is higher. For $M_{j}=2.5$, for example, the ratio of static pressure at the beginning of the cutout to the ambient pressure is 5.3 , in comparison with 2.3 in the previous case. The increased underexpansion generates stronger pairs of counter-rotating streamwise vortices and in turn results in more energetic mixing. As in the previous cases, the vortex pairs interact in nozzle $\mathrm{RS}$ at $x / D_{\mathrm{eq}}=2$. This interaction results in reduced growth rate after the interaction as shown in Fig. 3a. In the underexpanded flow regime, the mixing areas of nozzle $\mathrm{RS}$ at $x / D_{\mathrm{eq}}=2$ and 8 show 30 and $25 \%$ increases over that of a nozzle with the same cutout located on the splitter plate. ${ }^{1}$

For nozzle RC, a mushroom-type pair of streamwise vortices roll up farther away from the jet center when compared to that of a nozzle with the same cutout located on the splitter plate. ${ }^{1}$ At both $x / D_{\mathrm{e}}=2$ and 8, the normalized mixing area of nozzle $R C$ was increased about $20 \%$ over that of a nozzle with the same cutout located on the splitter plate. ${ }^{1}$ The increased spanwise pressure gradient on the contoured nozzle block with a cutout seemed to generate a stronger mushroomtype pair of counter-rotating streamwise vortices. By the pumping

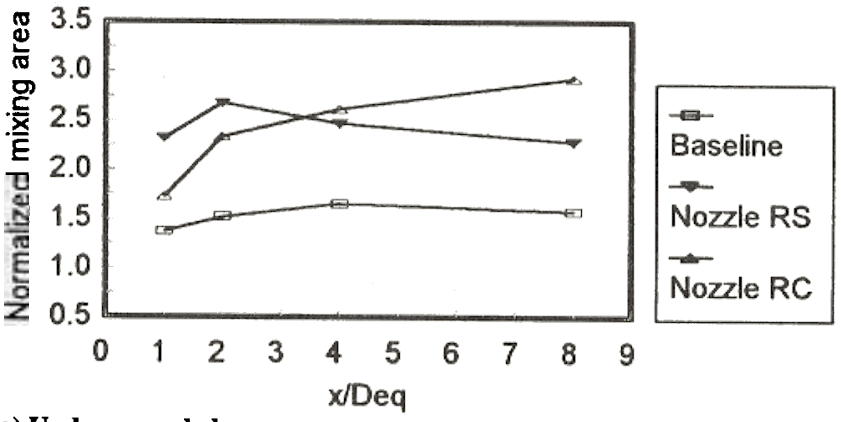

a) Underexpanded

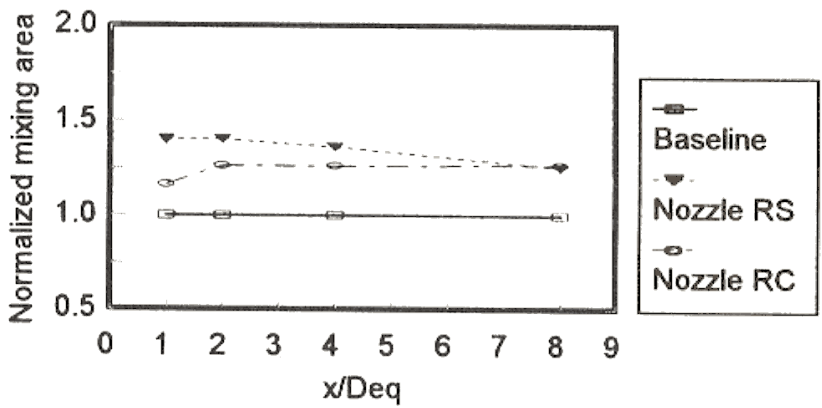

b) Ideally expanded

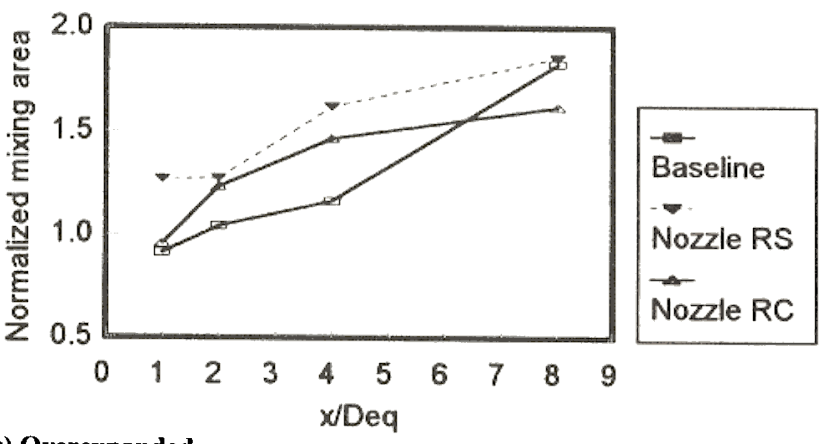

c) Overexpanded

Fig. 3 Normalized mixing areas with downstream location. 
action of this pair of vortices, a significant ejection of the jet air into the ambient air was observed at all downstream locations. Similar to previous work, ${ }^{2}$ this favorable pumping action of mushroom-type vortices resulted in a continuous increase in mixing as shown in Fig. 3a. As in the previous experiments, ${ }^{2}$ nozzle RS, which generates a kidney-type pair of streamwise vortices, showed better mixing in the near field, whereas nozzle RC, which generates a mushroomtype pair of streamwise vortices, showed better mixing at locations farther downstream, as shown in Fig. 3a.

\section{Ideally Expanded Case $\left(M_{j}=2.0\right)$}

Significant jet cross-sectional development is observed for this flow condition by the cutout on the contoured nozzle block. With this nozzle configuration, the jet cross-sectional developments with downstream location for nozzles RS and RC are similar to those in the underexpanded flow regime of $M_{j}=2.2$ with similar cutouts as in previous experiments. This is expected because the ratio of the static pressure at the beginning of the cutout to the ambient pressure is 2.4 , in comparison with 1.0 in the previous cases with cutouts on the splitter plate or on the nozzle extension. This underexpansion generates a surface pressure gradient around the cutout, which is a necessary condition for streamwise vorticity generation. The two pairs of counter-rotating streamwise vortices are similar to those in the $M_{j}=2.5$ case, although not strong enough to deform the jet cross section dramatically.

Mixing areas at $x / D_{\mathrm{ey}}=2$ and 8 increased about $20 \%$ when they are compared with those for nozzles with similar cutouts on the splitter plate. ${ }^{1}$ Nozzle RS shows better mixing in the near field as in the $M_{j}=2.5$ case.

\section{Overexpanded Case $\left(M_{j}=1.75\right)$}

With the cutout on the contoured nozzle block, the jet crosssectional development is significantly altered for locations far downstream. The jet cross sections of nozzle RS and RC show an axis switching, as in the baseline nozzle, by the $x / D_{\mathrm{eq}}=8$ location for the present cases. On the other hand, only the baseline nozzle showed an axis switching by this location in the previous cases. ${ }^{1}$ From the instantaneous images, the degree of flapping motion of mixing layers can be inferred. ${ }^{4}$ In the previous cases, ${ }^{2,4}$ most of the cutouts significantly reduced the flapping motion. However, the present cutout did not significantly change the flapping motion. The unaltered flapping motion is most likely responsible for the enhanced mixing.

Nozzle RS shows approximately $60 \%$ increased mixing at $x / D_{\text {eq }}=8$ when compared with that of a nozzle with a similar cutout on the splitter plate, ${ }^{1}$ although it shows a little reduced mixing at $x / D_{\mathrm{eq}}=2$. Nozzle RC shows a reduced mixing level at $x / D_{\mathrm{eq}}=2$ and about the same mixing level at $x / D_{\text {eq }}=8$ when it is compared with that of a nozzle with a similar cutout on the splitter plate. ${ }^{1}$ Contrary to the previous cases, the growth rates of mixing area for both nozzles RS and RC are positive all the way up to $x / D_{\mathrm{eq}}=8$. As mentioned earlier, the unaltered jet flapping motion is most likely related to the enhanced mixing.

\section{Conclusions}

A rectangular nozzle with a cutout on the contoured nozzle block showed higher mixing levels than previous experiments with the cutouts either on the splitter plate in a half nozzle or on the extension plates in a full nozzle. Except for the increased mixing level, the overall development of jet cross sections with downstream locations and the role of streamwise vortices remained similar to those of previous experiments with the cutouts on a splitter plate or a nozzle extension plate. The role of streamwise vortices in the jet development of the ideally expanded case seemed to be similar to those in the underexpanded case.

In the overexpanded flow regime, the present nozzle with a cutout on a contoured nozzle block showed about the same level of flapping motion as the baseline nozzle, whereas nozzles with cutouts on the splitter or on the extension in the previous experiments showed reduced flapping. The unaltered flapping motion of nozzles with a cutout resulted in positive growth rates all the way up to $x / D_{\mathrm{eq}}=8$, whereas the growth rates of nozzles with cutouts on nozzle extensions were negative at locations far downstream. Therefore, a nozzle with a cutout on a contoured nozzle block would perform better in mixing at all flow conditions. Note that in the previous experiments with the cutouts either on the splitter plate in a half nozzle or on the nozzle extension in a full nozzle, there was no thrust loss associated with the cutouts. However, no thrust measurements were performed for the present cutouts.

\section{Acknowledgments}

The support of this research by NASA John H. Glenn Research Center at Lewis Field (with K. Zaman) and by the Air Force Office of Scientific Research (with S. Walker and T. Beutner) is very much appreciated.

\section{References}

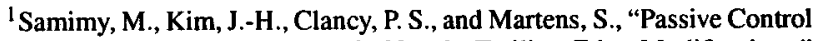
of Supersonic Rectangular Jets via Nozzle Trailing-Edge Modifications," AIAA Journal, Vol. 36, No. 7, 1998, pp. 1230-1239.

${ }^{2}$ Kim, J.-H., and Samimy, M., "Mixing Enhancement via Nozzle Trailing Edge Modifications in a High Speed Rectangular Jet," Physics of Fluids, Vol. 11, No. 9, 1999, pp. 2731-2742.

${ }^{3}$ Kerechanin, C., Samimy, M., and Kim, J.-H. "Effects of Nozzle TrailingEdge Modifications on Noise Radiation in Supersonic Rectangular Jets," AIAA Paper 2000-0086, Jan. 2000.

${ }^{4} \mathrm{Kim}, \mathrm{J}$.-H., "An Experimental Study of Mixing and Noise in a Supersonic Rectangular Jet with Modified Trailing Edges," Ph.D. Dissertation, Dept. of Mechanical Engineering, Ohio State Univ., Columbus, OH, 1998.

J. P. Gore Associate Editor

\section{Insensitivity of Unsteady Vortex Interactions to Reynolds Number}

\author{
Caroline Lambert* and Ismet Gursul ${ }^{\dagger}$ \\ University of Bath, \\ Bath, England BA2 7AY, United Kingdom
}

\section{Introduction}

$\mathbf{S}$ EVERAL investigations have revealed that the vortex breakdown location over delta wings exhibits quasi-periodic oscillations along the axis of the vortices due to an interaction between the two leading-edge vortices. These observations were made by flow visualization in water-tunnel facilities at low Reynolds numbers, and the issue arises as to whether this is a low-Reynolds-number phenomenon. In this Note, by using two-point unsteady surface pressure measurements in a wind tunnel, it is shown that this phenomenon exists at much higher Reynolds numbers.

The antisymmetric motion of breakdown locations for left and right vortices (Fig. 1) can be demonstrated by studying the difference between the breakdown locations $\left(X_{\text {left }}-X_{\text {right }}\right) / c$ and the average breakdown location $\left(X_{\text {left }}+X_{\text {right }}\right) / 2 c$. The spectra of these are shown in Fig. 2 for $\Lambda=75 \mathrm{deg}$ and $\alpha=42 \mathrm{deg}$ (taken from Ref. 1). It is seen that most of the energy is concentrated in the difference and that there is a dominant peak corresponding to the quasi-periodic antisymmetric oscillations. Experiments on the nature and source of these oscillations as well as the effect of angle of attack and sweep angle are reported in detail in Ref. 2. Similar observations of the quasi-periodic oscillations of breakdown location were also made by others ${ }^{3-7}$ by using flow visualization in water tunnels. The range of Reynolds number in these water-tunnel experiments and the frequency of the organized motion are shown in Table 1. Note that these oscillations were observed at Reynolds

Received 27 August 1999; revision received 17 November 1999; accepted for publication 22 December 1999. Copyright (C) 2000 by Caroline Lambert and Ismet Gursul. Published by the American Institute of Aeronautics and Astronautics, Inc., with permission.

* Graduate Student, Department of Mechanical Engineering.

${ }^{\dagger}$ Reader, Department of Mechanical Engineering. Senior Member AIAA. 